\title{
Metabolomic analysis of blood plasma from patients with mesial temporal lobe epilepsy: a search for biomarkers of drug resistance
}

\author{
Alexandre B. Godoi ${ }^{*}$ Amanda M. Canto, Amanda Donatti, Douglas C. Rosa, Marina K. Alvim, Clarissa L. Yasuda, \\ Mariana Martin, Melissa Quintero, Fernando Cendes, Ljubica Tasic, Iscia Lopes-Cendes.
}

\begin{abstract}
Mesial temporal lobe epilepsy (MTLE) stands out among the different types of epilepsy due to its prevalence and the high rate of resistance to the treatment with antiepileptic drugs (AEDs). Because of the challenge to predict which patients will respond to drug treatments, alternative therapies to seizures, such as epilepsy surgery, may take many years to be indicated. Thereby, the search for new biomarkers, capable of predicting which pateitns witll be AED resistance is necessary. The metabolomic approach has many advantages over other molecular techniques, such as greater proximity of the metabolites to the phenotype and high sensitivity and specificity in the detection of these analytes. Therefore, the main objective of the present sutidy is to use metabolomics to find biomarkers of AED resistance in patients with MTLE.
\end{abstract}

\section{Keywords:}

Mesial Temporal Lobe Epilepsy, Metabolomics, Proton Nuclear Magnetic Resonance (NMR- $\left.H^{1}\right)$.

\section{Introduction}

Mesial temporal lobe epilepsy (MTLE) is the most frequent type of epilepsy in adults ${ }^{1}$. It is characterized by the presence of recurrent simple or complex partial seizures, accompanied by secondary generalization ${ }^{2}$. In addition, about $30 \%$ of patients with MTLE do not respond to the prescribed antiepileptic drug $(A E D)^{3}$. In these patients, it is common to find the association with a specific neuropathological lesion named mesial temporal sclerosis (MTS) ${ }^{4}$. For these patients with MTS and AED resistance, surgical intervention may be an alternative procedure for treatment, and it has a good chance of promoting a significant improvement in the frequency and severity of seizures ${ }^{5}$. However, surgical intervention is not always performed early enough, resulting in a long period of exposure to the side effects of the AEDs and the high occurrence of seizures ${ }^{3}$. In this sense, an investigation of the metabolic profile can offer not only better tools to identify which patients are resistant to AED therapy but also help in the identification of metabolic factors associated with the resistance to AED therapy, thus, accelerating and optimizing the indication of epilepsy surgery. Metabolomics uses several analytical techniques to provide data on the metabolic profile through various physical-chemical principles. One of the most widely used methods is the proton nuclear magnetic resonance spectrometry ( $\left.{ }^{1} \mathrm{H}-\mathrm{NMR}\right)$. The use of ${ }^{1} \mathrm{H}-\mathrm{NMR}$ in the context of metabolomics is supported by its high reproducibility, without the need for complex sample preparation, and associated with the chemometric analysis could provide us specific metabolites that characterize the profile of drug resistance to the AEDs in MTLE ${ }^{6}$.

\section{Results and Discussion}

This is an ongoing project, and to date, we have analyzed twenty-two samples (22), five controls (5), nine MTLE patients resistant to AED treatment (9), and eight MTLE patients who are responsive to AED treatment (8). The blood plasma was prepared and analyzed in a $600-\mathrm{MHz}$ BurkerAscend $^{\mathrm{TM}}$ spectrometer (Chemistry Institute UNICAMP). The obtained raw spectra were processed to gauge them and analyzed. The responsive patients revealed stronger signals at spectral regions $\delta 7.76$ and $\delta 7.16$, related to the aromatic amino acids: tryptophan and tyrosine, respectively, and a signal at 83.97 , related to creatine when compared to resistant patients and controls. In addition, we also found one stronger signal in resistant patients at spectral region $\delta 7.28$, related to phenylalanine, when compared to responsive patients and controls; and one stronger signal in controls at spectral region $\delta 1.94$, related to acetate, when compared to both groups of patients.

\section{Conclusions}

We report here for the first time, preliminary results of metabolomic analysis of plasma samples from patients with MTLE, which present different responses to AED treatment. Due to the limited number of samples studied until now, we cannot provide a specific metabolic profile of the groups analyzed. However, our preliminary data already indicates that there may be differences in the plasma metabolomic profile of the groups studied.

\section{Acknowledgement}

This project is part of the Brazilian Institute of Neuroscience and Neurotechnology (BRAINN) and is supported by FAPESP.

\footnotetext{
${ }^{1}$ Engel, J., Jr. Introduction to temporal lobe epilepsy. Epilepsy Res, 1996; ${ }^{2}$ Blumcke, I.; Zuschratter, W.; Schewe, J.C.; Suter, B.; Lie, A.A. Rieder, B.M.; Meyer, B.; Schramm, J.; Elger, C.E.; Wiestler, O.D. Cellular pathology of hilar neurons in Ammon's horn sclerosis. 1999. J Comp Neurol 414, 437453

${ }^{3}$ Yassuda, C.L.; Cendes, F. Neuroimaging for the prediction of response to medical and surgical treatment in epilepsy. Expert Opin Med Diagn. Jul. 2012, 6(4):295-308,

${ }^{4}$ Blumcke I.; Beck, H.; Lie, A.A.; Wiestler, O.D. Molecular neuropathology of human mesial temporal lobe epilepsy. Epilepsy Res. 1999, Sep;36(2 3):205-23. Review;

${ }^{5}$ Schramm, J.; Kral, T.; Grunwald, T.; Blumcke, I. Surgical treatment for neocortical temporal lobe epilepsy: Clinical and surgical aspects and seizure outcome. J. Neurosurg. 2001, 94, 33-42;

${ }^{6}$ Gebregiworgis, T.; Powers, R. Application of NMR Metabolomics to Search for Human Disease Biomarkers. Combinatorial Chemistry \& High Throughput Screening, 2012.
} 